\title{
Unenhanced computed tomography for non-invasive diagnosis of hepatic steatosis with low tube potential protocol
}

\author{
Yunjung Choi ${ }^{1 \wedge}$, Dong Kyun $\mathrm{Kim}^{1 *}$, Seo Yeon Youn ${ }^{1 \wedge}$, Hokun Kim ${ }^{1 \wedge}$, Joon-Il Choi ${ }^{1,2 *}$ \\ ${ }^{1}$ Department of Radiology, Seoul St. Mary's Hospital, College of Medicine, The Catholic University of Korea, Seoul, Republic of Korea; ${ }^{2}$ Catholic \\ Cancer Research Institute, College of Medicine, The Catholic University of Korea, Seoul, Republic of Korea
}

Contributions: (I) Conception and design: JI Choi; (II) Administrative support: SY Youn, H Kim; (III) Provision of study materials or patients: Y Choi, DK Kim; (IV) Collection and assembly of data: Y Choi, DK Kim; (V) Data analysis and interpretation: DK Kim, JI Choi; (VI) Manuscript writing: All authors; (VII) Final approval of manuscript: All authors.

*These authors contributed equally to this work as co-corresponding authors.

Correspondence to: Joon-Il Choi, MD. Department of Radiology, Seoul St. Mary's Hospital, College of Medicine, The Catholic University of Korea, 222 Banpo-daero, Seocho-gu, Seoul 06591, Republic of Korea. Email: dumkycji@gmail.com.

Background: Lowering $\mathrm{kVp}$ affects the image contrast and computed tomography (CT) attenuation values of low $\mathrm{kVp} C T$ is different from those of conventional $120-\mathrm{kVp}$ scans. The purpose of this study is to determine the diagnostic performance and to establish the reference range of low- $\mathrm{kVp}$ unenhanced CT for the assessment of hepatic steatosis in liver transplantation donors using magnetic resonance (MR) spectroscopy as a reference standard.

Methods: This retrospective study included 165 potential donors (male:female $=114: 51,36.5 \pm 12.0$ years old) who underwent $100-\mathrm{kVp}$ single-slice unenhanced CT scan and MR spectroscopy. The difference between hepatic and splenic attenuation $\left(\mathrm{CT}_{\mathrm{L}-\mathrm{S}}\right)$ and liver-to-spleen attenuation ratio $\left(\mathrm{CT}_{\mathrm{L} / \mathrm{S}}\right)$ were calculated. Reference standard was the fat signal fraction measured by MR spectroscopy. Limits of agreement between CT measurements and the reference standard were calculated. Areas under receiver operating characteristic curves (AUROCs) of $\mathrm{CT}_{\mathrm{L}-\mathrm{S}}$ and $\mathrm{CT}_{\mathrm{L} / \mathrm{S}}$ were compared for the diagnosis of moderate to severe steatosis. Cutoff values of $\mathrm{CT}_{\mathrm{L}-\mathrm{S}}$ and $\mathrm{CT}_{\mathrm{L} / \mathrm{S}}$ that provided a balance between sensitivity and specificity and the highest specificity using the lower limit of the reference range were calculated.

Results: Eighty-seven subjects had a non-steatotic liver. Sixty-one subjects had mild steatosis and 17 subjects had moderate to severe steatosis based on MR spectroscopy. $\mathrm{CT}_{\mathrm{L}-\mathrm{S}}$ and $\mathrm{CT}_{\mathrm{L} / \mathrm{S}}$ values were negatively correlated with the fat signal fraction $(\mathrm{P}<0.001)$ and limits of agreement were $-8.4 \%$ to $8.4 \%$ for $\mathrm{CT}_{\mathrm{L}-\mathrm{S}}$ and $-9.6 \%$ to $9.6 \%$ for $\mathrm{CT}_{\mathrm{L} / \mathrm{S}}$. AUROCs of $\mathrm{CT}_{\mathrm{L}-\mathrm{S}}$ and $\mathrm{CT}_{\mathrm{L} / \mathrm{S}}$ for diagnosing moderate to severe steatosis were 0.956 and 0.957 , respectively. Cut-off values of $\mathrm{CT}_{\mathrm{L}-\mathrm{S}}$ and $\mathrm{CT}_{\mathrm{L} / \mathrm{S}}$ for diagnosis of moderate to severe steatosis by the Youden index were $-0.5 \mathrm{HU}$ for $\mathrm{CT}_{\mathrm{L}-\mathrm{S}}$ and 0.99 for $\mathrm{CT}_{\mathrm{L} / \mathrm{S}}$. Reference ranges of non-steatotic liver were -6.90 to $31.40 \mathrm{HU}$ for $\mathrm{CT}_{\mathrm{L}-\mathrm{S}}$ and 0.89 to 1.77 for $\mathrm{CT}_{\mathrm{L} / \mathrm{S}}$. Using $-6.9 \mathrm{HU}$ for $\mathrm{CT}_{\mathrm{L}-\mathrm{S}}$ and 0.89 for $\mathrm{CT}_{\mathrm{L} / \mathrm{s}}$ as cut-off values, the sensitivity and specificity for diagnosing moderate to severe steatosis were $70.59 \%$ and 90.54\% $\left(\mathrm{CT}_{\mathrm{L}-\mathrm{S}}\right)$ and $76.47 \%$ and $90.54 \%\left(\mathrm{CT}_{\mathrm{L} / \mathrm{S}}\right)$, respectively.

Conclusions: Measurements from a low $-\mathrm{kVp}$ unenhanced CT scan were negatively correlated with the degree of hepatic steatosis. Low-kVp unenhanced CT is a robust technique with reduced radiation exposure for diagnosing moderate to severe hepatic steatosis.

Keywords: Liver; steatosis; computed tomography (CT); magnetic resonance spectroscopy (MRS)

\footnotetext{
^ ORCID: Yunjung Choi, 0000-0003-0983-0441; Dong Kyun Kim, 0000-0003-3092-3810; Seo Yeon Youn, 0000-0002-7692-3413; Hokun Kim, 0000-0003-0884-7946; Joon-Il Choi, 0000-0003-0018-8712.
} 
Submitted May 06, 2021. Accepted for publication Sep 18, 2021.

doi: 10.21037/qims-21-474

View this article at: https://dx.doi.org/10.21037/qims-21-474

\section{Introduction}

Non-alcoholic fatty liver disease (NAFLD) is the most common liver parenchymal disease and an important health issue because NAFLD is associated not only with liver diseases such as non-alcoholic steatohepatitis, cirrhosis, and hepatocellular carcinoma, but also various systemic problems including cardiovascular and metabolic disease (1-5). Furthermore, hepatic steatosis, especially macrovesicular hepatic steatosis, affects liver transplantation outcomes $(6,7)$. In living donor liver transplantation (LDLT), the degree of hepatic steatosis affects both recipient outcomes and donor safety because hepatic steatosis increases the incidence of graft failure in recipients and impairs hepatic regeneration in donors (8-10).

Although liver biopsy is regarded to be the gold standard method for establishing a diagnosis of hepatic steatosis, various imaging methods and biochemical evaluations are widely used to evaluate hepatic steatosis. Magnetic resonance imaging (MRI) techniques including two-point Dixon dual echo imaging, magnetic resonance spectroscopy (MRS) and MRI proton density fat fraction (MRIPDFF) have diagnostic value similar to that of histologic examination for assessment of hepatic steatosis (11-16). Meanwhile dual echo imaging is mainly a qualitative tool, MRS and MRI-PDFF are quantitative tools.

Previous studies reported that unenhanced computed tomography (CT) had a high specificity for diagnosing macrovesicular steatosis $30 \%$ or greater and could help avoid unnecessary biopsy in potential LDLT donors (7,17-20). However, previous research results were derived from unenhanced CT scans obtained at $120-\mathrm{kVp}$ in the early 2000s. Since the 2010s, tube potential selection techniques have been widely adopted to reduce radiation exposure, and low-kVp images have become increasingly popular. As a consequence, abdominal CT scans using $100-\mathrm{kVp}$ or $80-\mathrm{kVp}$ are generally obtained, especially in Asian countries where patients tend to be small in size. In our institution, abdominal CT images for LDLT donors are usually obtained at $100-\mathrm{kVp}$ to reduce radiation exposure. The unenhanced scan is obtained as a single slice image covering the spleen and liver to further reduce radiation exposure. Lowering $\mathrm{kVp}$ affects the image contrast and $\mathrm{CT}$ attenuation values of low $\mathrm{kVp}$-CT is different from those of conventional $120-\mathrm{kVp}$ scans. To the best of our knowledge, no prior study has evaluated the diagnostic performance or reference range of low-kVp CT attenuation values for evaluating hepatic steatosis.

Therefore, our aim in the current study was to investigate whether CT indices measured on a single slice unenhanced image obtained at a low-kVp were different from those obtained at $120-\mathrm{kVp}$ using MRS as the standard of reference.

We present the following article in accordance with the STARD reporting checklist (available at https://dx.doi. org/10.21037/qims-21-474).

\section{Methods}

\section{Study population}

The study was conducted in accordance with the Declaration of Helsinki (as revised in 2013). The study was approved by the institutional review board of the Catholic University of Korea (No. KC21RASI0263) and individual consent for this retrospective analysis was waived. This was an exploratory research, and the comparison of diagnostic performance of low-kVp CT to full dose CT was not the purpose of the study. Therefore, we did not perform power analysis. The institutional database was searched for liver donor protocol CT for LDLT between January 2014 and May 2020.

Inclusion criteria for this study were as follows: (I) potential liver donor for living-donor liver transplantation; (II) subject underwent liver donor protocol CT including low-kVp (100-kVp) single-slice unenhanced CT covering the part of liver and spleen, and (III) the subject underwent MRS to evaluate hepatic steatosis within 7 days of liver CT examination. Exclusion criteria were as follows: (I) spleen not covered by low-kVp single-slice unenhanced CT, and (II) poor image quality of the unenhanced CT scan due to severe motion artifacts. A total of 249 consecutive, potential donors for LDLT underwent liver CT and/or MRI for preoperative evaluation. Among them, 84 donor candidates were excluded from the study because a low-kVp $(100-k V p)$ single-slice unenhanced CT scan was not taken $(n=60)$, the spleen was not included in the single slice unenhanced CT scan $(n=12)$, or MRS was not performed $(n=12)$. The remaining 165 donor candidates were enrolled in this study 


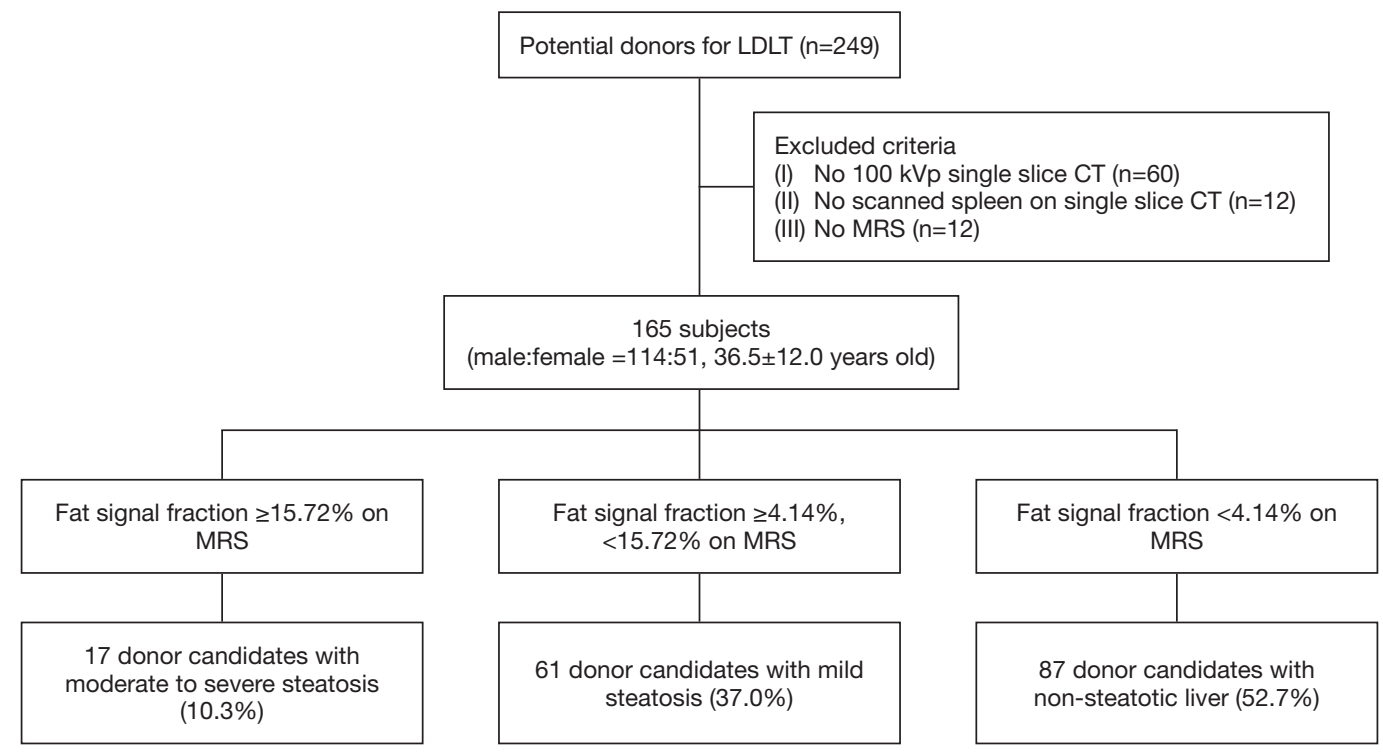

Figure 1 Diagram showing donor candidate enrollment. LDLT, living donor liver transplantation; CT, computed tomography; MRS, magnetic resonance spectroscopy.

(male:female $=114: 51$, mean age $36.5 \pm 12.0$ years) (Figure 1$)$.

\section{Image acquisition techniques}

Liver donor protocol CT scans were performed using a multi-detector low (64 channels or higher) CT scanner (Siemens SOMATOM definition AS+, Siemens Sensation 64, Siemens SOMATOM Force, or Siemens SOMATOM definition Edge, Siemens Healthineers, Erlangen, Germany) in all subjects. A single-slice low- $\mathrm{kV}$ p unenhanced scan covering the liver and spleen in addition to early arterial phase and portal venous phase images were obtained. Scan and reconstruction parameters for low- $\mathrm{kV}$ p unenhanced CT with a single slice were beam collimation of $128 \mathrm{~mm} \times 0.6 \mathrm{~mm}$, beam pitch of 1 , gantry rotation time of 0.5 seconds, tube voltage of $100-\mathrm{kVp}$, and tube current of $20 \mathrm{mAs}$. To assess CT radiation dose, the dose length product (DLP) was calculated as $0.67 \mathrm{mGy} \cdot \mathrm{cm}$.

In all enrolled subjects, unenhanced liver MRI [axial T2weighted image with fast spin echo and single-shot halfFourier turbo spin echo, axial in-and-opposed phase T1 gradient recalled echo (GRE) images], 2D/3D magnetic resonance cholangiopancreatography to evaluate bile duct anatomy, and multiecho MRS to evaluate hepatic steatosis were performed. MRI was performed using one of two 3-Tesla (3T) scanners (Siemens Verio or Siemens MAGNETOM Vida, Siemens Healthineers, Erlangen,
Germany). MRS was conducted using single-voxel technique called HISTO with stimulated echo acquisition mode (STEAM). A $3 \times 3 \times 3 \mathrm{~cm}^{3}$ voxel was placed in the hepatic parenchyma of the right hemiliver to avoid hepatic vessels, bile ducts, or focal hepatic lesions. During a single 15 second breath hold, five STEAM spectra were generated at multiple echo times (TEs) of 12, 24, 36, 48, and $72 \mathrm{~ms}$. We used a long repetition time (TR) of 3,000 $\mathrm{ms}$ to minimize $\mathrm{T} 1$ weighting and high-speed $\mathrm{T} 2$ correction to prevent over-evaluating proton density fat fraction (21). The fat signal fraction and R2 water value can be calculated using this approach (Figure 2). In this study, we adopted fat signal fractions of $4.14 \%$ and $15.72 \%$ to classify any degree of hepatic steatosis (macrovesicular steatosis of less than $5 \%$ at histology) and moderate to severe hepatic steatosis (at least 30\% macrovesicular steatosis at histology), respectively. These cut-off values of the fat signal fraction were based on the results of a previous study that used $3 \mathrm{~T}$ multiecho MRS with similar techniques (22). We adopted MRS as the reference standard because (I) liver biopsy was performed in only part of the subjects and (II) multiecho MRS was reported to be closely correlated to pathology results for evaluating hepatic steatosis (13-15).

\section{Data analysis}

For each candidate, hepatic attenuation (L) and splenic 

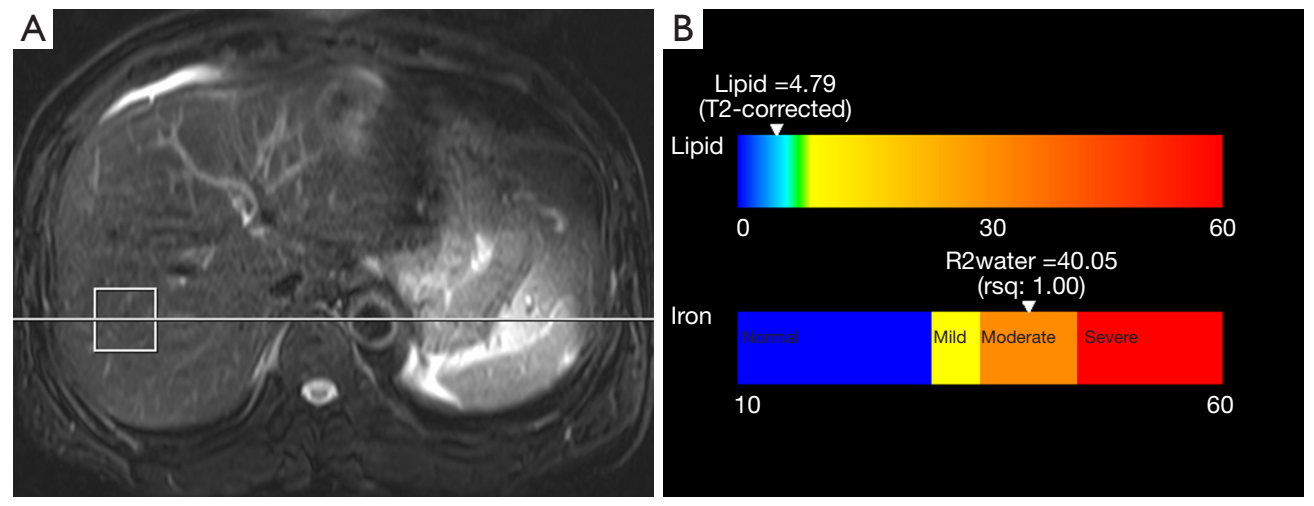

Figure 2 Magnetic resonance spectroscopy to measure the fat signal fraction. (A) A $3 \times 3 \times 3 \mathrm{~cm}^{3}$ voxel was located in the hepatic parenchyma of the right hemiliver to avoid hepatic vessels; (B) upper color bar shows the T2-corrected fat signal fraction (4.79\% in this case). Lower color bar is for R2 water to evaluate iron deposition.

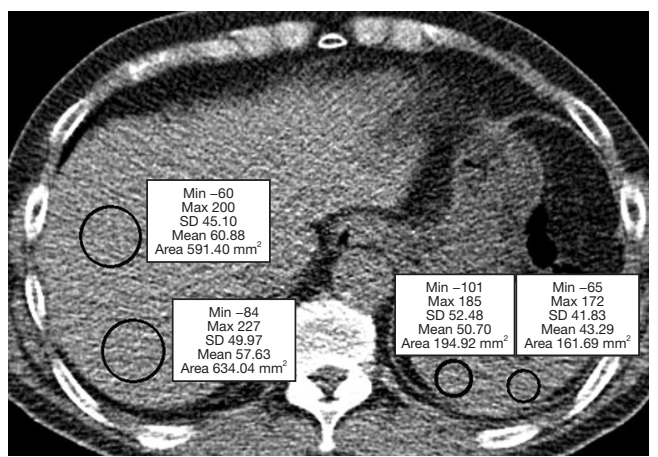

Figure 3 CT attenuation measurement on a low-kVp, singleslice unenhanced CT scan. Liver parenchyma attenuation was measured in two regions of interest (ROIs) of the right hemiliver. An ROI was placed in two different segments. To measure spleen attenuation, two ROI were placed in the spleen, regardless of the anatomical part of the spleen. Liver and spleen attenuation values in the two ROIs were averaged. CT, computed tomography; SD, standard deviation.

attenuation (S) were measured in a low- $\mathrm{kVp}$, single-slice unenhanced scan. $\mathrm{L}$ was measured as the average of the attenuation values of two regions of interest (ROIs) in Hounsfield unit (HU). ROIs were placed in two different segments in the right hemiliver. S was measured as the average of measurements in $\mathrm{HU}$ at two different ROIs in the spleen, regardless of underlying spleen anatomy (Figure 3). A senior resident of radiology department drew the ROIs and he/she was blinded to the clinical information and results of MRS or pathological examination for hepatic steatosis. Two different liver attenuation indices, the difference between hepatic and splenic attenuation $\left(\mathrm{CT}_{\mathrm{L}-\mathrm{S}}\right)$ and the liver-tospleen attenuation ratio $\left(\mathrm{CT}_{\mathrm{L} / \mathrm{S}}\right)$ were calculated to quantify the degree of hepatic steatosis (17). For the evaluation of reproducibility of ROI measurement, a junior resident drew the ROIs again and we compared the mean values of attenuation indices to first measurement. We used first measurement by a senior resident for further analyses.

Linear regression analyses were performed between the two liver attenuation indices derived from CT measurements and the fat signal fraction derived from MRS to derive regression equations. Furthermore, limits of agreement between steatosis estimated by the regression equation and the fat signal fraction estimated by MRS were calculated for both CT attenuation indices using BlandAltman plots. To compare the diagnostic performance of the two CT attenuation indices for the diagnosis of subjects with moderate to severe hepatic steatosis, receiver operating characteristic (ROC) analyses were performed and the areas under the ROC curves (AUROCs) and their 95\% confidence intervals (CIs) were calculated and compared for both CT attenuation indices.

$\mathrm{CT}_{\mathrm{L}-\mathrm{S}}$ and $\mathrm{CT}_{\mathrm{L} / \mathrm{S}}$ cut-off values were used to diagnose moderate to severe hepatic steatosis to (I) provide a balance between sensitivity and specificity calculated by the Youden index and (II) the determine the highest specificity based on the lower limit of the reference range as determined by the Clinical and Laboratory Standards Institute (CLSI) guideline C28-A3. Cut-off values to balance sensitivity and specificity were calculated by choosing the value that was nearest to the left corner of the ROC curve to minimize false positives and negatives. Using these cut-off values, 

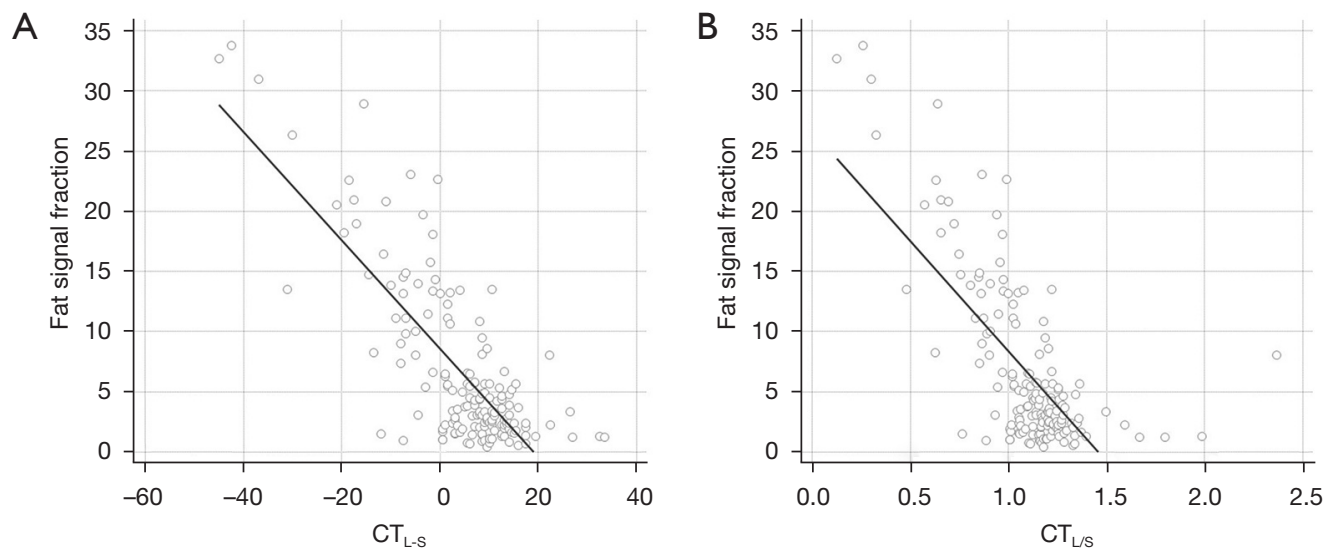

Figure 4 Scatter plots showing the results of linear regression analyses. (A) Scatterplots showing the results of linear regression analyses between the fat signal fraction and $\mathrm{CT}_{\mathrm{L}-\mathrm{S}}$ (the difference between hepatic and splenic attenuation). Linear lines are best-fit lines of the linear regression equations; (B) scatterplots showing the results of linear regression analyses between the fat signal fraction and $\mathrm{CT}_{\mathrm{L} / \mathrm{S}}$ (the liver-tosplenic attenuation ratio). Linear lines are best-fit lines of the linear regression equations. CT, computed tomography.

sensitivity, specificity, and 95\% CIs were calculated and compared between the two CT attenuation indices. A reference range was also calculated using the method published in CLSI guideline C28-A3 to determine reference intervals of quantitative clinical laboratory tests (18). CLSI guideline $\mathrm{C} 28-\mathrm{A} 3$ is a guideline for the reference interval, i.e., values for healthy population, of medical assays and the lower and upper limits of the reference range were estimated as the $2.5 \%$ and $97.5 \%$, respectively of the distribution in the reference population. This guideline is the most widely-used source of reference in this area and adopted by the statistical software packages. Calculation of the cut-off values based on the lower limit of the reference range by the CLSI guideline C28-A3 has two advantages: (I) to maximize specificity (97.5\%, theoretically) and (II) to reduce the influence of the subject population because ROC based cut-off values can be heavily influenced by the distribution of the severity of steatosis in the study population (23). We also checked the normality of values of each CT index using the Shapiro-Wilks test because CLSI guidelines recommend that the reference population follow a Gaussian distribution. Using these cut-off values, we calculated and compared sensitivity and specificity for any degree of steatosis ( $\geq 4.14 \%$ of the fat signal fraction) and moderate to severe steatosis $(\geq 15.72 \%$ of the fat signal fraction) using both CT attenuation indices.

To evaluate the reproducibility of ROI measurement, Lin's concordance correlation coefficient was calculated. The following classification was used for the level of agreement by concordance correlation coefficient: $<0.90$, poor; $\geq 0.90,<0.95$, moderate; $\geq 0.95,<0.99$, substantial; $\geq 0.99$, almost perfect agreement. To compare sensitivity and specificity, we used McNemar's test. AUROCs were compared by DeLong's method. IBM SPSS 24.0 for Windows (International Business Machines Corporation, New York, NY) and MedCalc 19.5.3 (MedCalc Software, Mariakerke, Belgium) were used for all statistical analyses. $\mathrm{P}<0.05$ was considered to indicate statistical significance.

\section{Results}

On MRS, the fat fraction signal ranged from $0.40 \%$ to $33.75 \%$ (mean $6.59 \% \pm 6.90 \%$, median $3.73 \%$ ) in the 165 donor candidates. Eighty-seven donor candidates $(52.7 \%$, $87 / 165)$ had a non-steatotic liver $(<4.14 \%$ of the fat signal fraction), 61 donor candidates $(37.0 \%, 61 / 165)$ had mild steatosis $(\geq 4.14 \%,<15.72 \%$ of the fat signal fraction), and 17 donor candidates $(10.3 \%, 17 / 165)$ had moderate to severe steatosis ( $\geq 15.72 \%$ of the fat signal fraction).

Lin's concordance correlation coefficients for liver attenuation, spleen attenuation, L-S and L/S were 0.956 , $0.905,0.936$ and 0.931 , respectively and the reproducibility of ROI measurement was moderate to substantial.

In linear regression analyses, fat signal fraction and CT attenuation indices were negatively correlated ( $r$ ho $=-0.603$, $\mathrm{P}<0.001$ for $\mathrm{CT}_{\mathrm{L}-\mathrm{S}}$ and rho $=-0.423, \mathrm{P}<0.001$ for $\mathrm{CT}_{\mathrm{L} / \mathrm{S}}$, rho is Spearman's correlation coefficient). Linear regression equations for fat signal fractions were $\left(-0.451 \times \mathrm{CT}_{\mathrm{L}-\mathrm{S}}\right)+$ 8.574 for $\mathrm{CT}_{\mathrm{L}-\mathrm{S}}$ and $\left(-18.310 \times \mathrm{CT}_{\mathrm{L} / \mathrm{S}}\right)+26.685$ for $\mathrm{CT}_{\mathrm{L} / \mathrm{S}}$ (Figure 4). Limits of agreement between the fat signal 

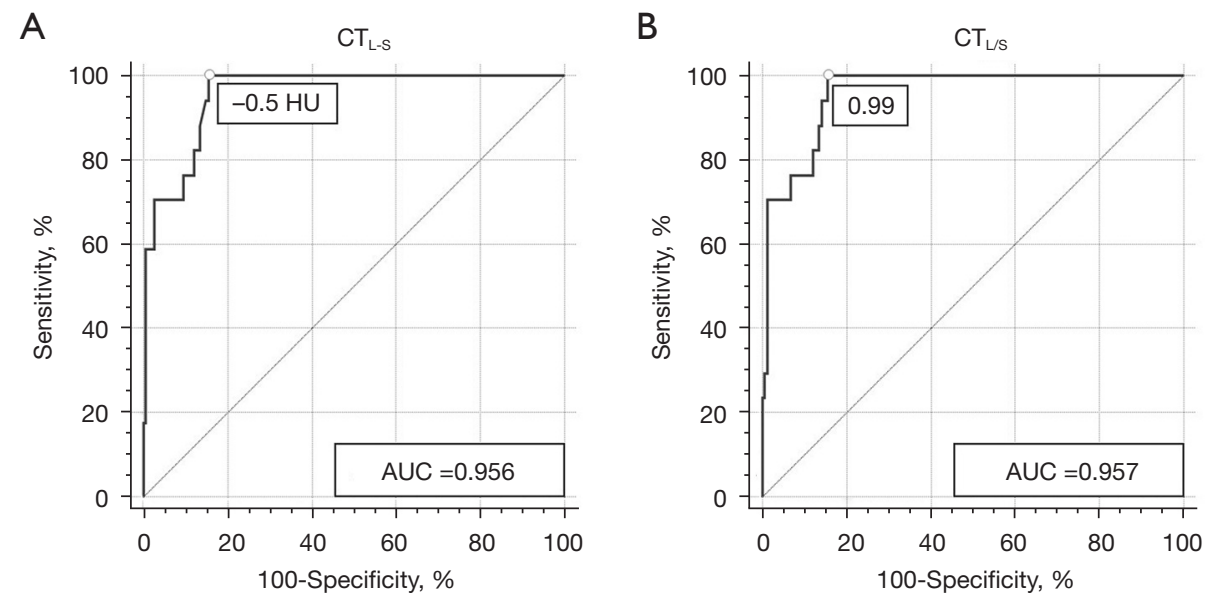

Figure 5 Receiver operating characteristics (ROC) curves for diagnosing moderate to severe hepatic steatosis $(\geq 15.72 \%$ of the fat signal fraction on magnetic resonance spectroscopy). (A) ROC curves for diagnosing moderate to severe hepatic steatosis using $\mathrm{CT}_{\mathrm{L}-\mathrm{S}}\left(\mathrm{the}_{\mathrm{B}}\right.$ difference between hepatic and splenic attenuation). Dots of each figure were the cut-off values by the Youden index; (B) ROC curves for diagnosing moderate to severe hepatic steatosis using $\mathrm{CT}_{\mathrm{L} / \mathrm{S}}$ (the liver-to-splenic attenuation ratio). Dots of each figure were the cut-off values by the Youden index. The areas under the ROC curves of the two CT indices were not significantly different (P=0.6314). AUC, area under the ROC curves; CT, computed tomography; HU, Hounsfield unit.

Table 1 Sensitivity, specificity, and positive and negative predictive values of $\mathrm{CT}_{\mathrm{L}-\mathrm{S}}$ and $\mathrm{CT}_{\mathrm{L} / \mathrm{S}}$ for diagnosing moderate to severe hepatic steatosis using cut-off values based on the Youden index

\begin{tabular}{lccc}
\hline Variables & CT L-S $_{\text {L }}$ & CT $_{\text {LS }}$ & P values \\
\hline Cut-off values & $-0.5 \mathrm{HU}$ & 0.99 & - \\
Sensitivity (\%) & $100.00(80.49-100.00)$ & $100.00(80.49-100.00)$ & $1.000^{*}$ \\
Specificity (\%) & $84.46(77.60-89.89)$ & $84.46(77.60-89.89)$ & $1.000^{*}$ \\
Positive predictive value (\%) & $42.50(33.67-51.83)$ & $42.50(33.67-51.83)$ & $1.000^{\#}$ \\
Negative predictive value (\%) & 100.00 & 100.00 & $1.000^{\#}$ \\
\hline
\end{tabular}

*, P values calculated using the McNemar's test; ", P values calculated using the Fisher's exact test. Numbers in parentheses are 95\% confidence intervals. $\mathrm{CT}_{\mathrm{L}-\mathrm{S}}$ : difference between liver and spleen attenuation on a low-kVp, single-slice unenhanced $\mathrm{CT}_{\mathrm{s}} \mathrm{scan}$; $\mathrm{CT}_{\mathrm{L} / \mathrm{s}}$ : ratio of liver and spleen attenuation on a low-kVp, single-slice unenhanced CT scan. CT, computed tomography.

fraction and values estimated from the regression equations were $-8.4 \%$ to $8.4 \%$ for $\mathrm{CT}_{\mathrm{L}-\mathrm{S}}$ and $-9.6 \%$ to $9.6 \%$ for $\mathrm{CT}_{\mathrm{L} / \mathrm{S}}$.

AUROCs of $\mathrm{CT}_{\mathrm{L}-\mathrm{S}}$ and $\mathrm{CT}_{\mathrm{L} / \mathrm{S}}$ for diagnosing moderate to severe steatosis were 0.956 (95\% CI: 0.912-0.982) and 0.957 (95\% CI: 0.914-0.986), respectively, with no significant difference $(\mathrm{P}=0.6314)$ (Figure 5). Cut-off values of $\mathrm{CT}_{\mathrm{L}-\mathrm{S}}$ and $\mathrm{CT}_{\mathrm{L} / \mathrm{S}}$ for diagnosis of moderate to severe hepatic steatosis by the Youden index were $-0.5 \mathrm{HU}$ and 0.99 , respectively. With these cut-off values, the sensitivity and specificity of $\mathrm{CT}_{\mathrm{L}-\mathrm{S}}$ and $\mathrm{CT}_{\mathrm{L} / \mathrm{S}}$ for diagnosing moderate to severe hepatic steatosis were the same: $100.0 \%$ sensitivity and $84.46 \%$ specificity (Table 1).
Reference ranges of $\mathrm{CT}_{\mathrm{L}-\mathrm{S}}$ and $\mathrm{CT}_{\mathrm{L} / \mathrm{S}}$ of donor candidates with non-steatotic liver were -6.90 to $31.40 \mathrm{HU}$ (mean $10.02 \pm 7.24 \mathrm{HU}$, median $10.00 \mathrm{HU}$ ) and 0.89 to 1.77 (mean $1.21 \pm 0.17$, median 1.19 ), respectively. We adopted the non-parametric percentile method of CLSI C28-A3 because the Shapiro-Wilks test rejected the normality of $\mathrm{CT}$ indices of donor candidates with nonsteatotic liver $\left(\mathrm{P}=0.0016\right.$ for $\mathrm{CT}_{\mathrm{L}-\mathrm{S}}$ and $\mathrm{P}<0.001$ for $\mathrm{CT}_{\mathrm{L} / \mathrm{S}}$, respectively). Using $-6.90 \mathrm{HU}$ for $\mathrm{CT}_{\mathrm{L}-\mathrm{S}}$ and 0.89 for $\mathrm{CT}_{\mathrm{L} / \mathrm{s}}$ as cut-off values, sensitivity and specificity of $\mathrm{CT}_{\mathrm{L}-\mathrm{S}}$ for any degree of hepatic steatosis were $29.49 \%$ and $97.70 \%$, respectively, while sensitivity and specificity of $\mathrm{CT}_{\mathrm{L} / \mathrm{S}}$ were 
Table 2 Sensitivity and specificity of $\mathrm{CT}_{\mathrm{L}-\mathrm{S}}$ and $\mathrm{CT}_{\mathrm{L} / \mathrm{S}}$ for diagnosing hepatic steatosis using cut-off values based on the lower limit of the reference range determined by CLSI guideline C28-A3

\begin{tabular}{|c|c|c|c|}
\hline Variables & $\mathrm{CT}_{\mathrm{L}-\mathrm{S}}$ & $\mathrm{CT}_{\text {LS }}$ & $P$ values \\
\hline \multicolumn{4}{|c|}{ Any degree of hepatic steatosis } \\
\hline Sensitivity (\%) & $29.49(19.70-40.89)$ & $30.77(20.81-42.24)$ & 1.000 \\
\hline Specificity (\%) & $97.70(91.94-99.72)$ & 97.70 (91.94-99.72) & 1.000 \\
\hline Sensitivity (\%) & $70.59(44.0-89.7)$ & 76.47 (50.1-93.2) & 1.000 \\
\hline Specificity (\%) & $90.54(84.6-94.7)$ & $90.54(84.6-94.7)$ & 1.000 \\
\hline
\end{tabular}

$P$ values were calculated using McNemar's test. Numbers in parentheses are $95 \%$ confidence intervals. $\mathrm{CT}_{\mathrm{L}-\mathrm{S}}$ : difference between liver and spleen attenuation on a low-kVp, single-slice unenhanced CT scan; $\mathrm{CT}_{\mathrm{L} / \mathrm{s}}$ : ratio of liver and spleen attenuation on a low-kVp, single-slice unenhanced CT scan; CLSI guideline C28-A3: the Clinical and Laboratory Standards Institute guideline for reference interval. CT, computed tomography; HU, Hounsfield unit.

$30.77 \%$ and $97.70 \%$, respectively. Using these same cut-off values, sensitivity and specificity for diagnosing moderate or severe steatosis were $70.59 \%$ and $90.54 \%$ for $\mathrm{CT}_{\mathrm{L}-\mathrm{S}}$, respectively, and $76.47 \%$ and $90.54 \%$ for $\mathrm{CT}_{\mathrm{L} / \mathrm{s}}$, respectively (Table 2). There were no adverse events related to CT scans or MRS.

\section{Discussion}

Although liver biopsy provides a definitive diagnosis of hepatic steatosis and is therefore considered the gold standard method, it is an invasive method that may result in complications. Furthermore, sampling bias cannot be avoided because only a small amount of tissue can be obtained by biopsy and examined by pathological evaluation, and pathologist subjectivity may influence the diagnosis (14-18). Imaging examinations such as ultrasonography, CT, and MRI are non-invasive and useful tools for diagnosing hepatic steatosis. Although MRI is the most accurate of these imaging modalities, unenhanced CT scans can be used for reliable qualitative diagnosis of macrovesicular steatosis of $30 \%$ or greater $(7,17,18)$. However, CT exposes patients and healthy individuals such as liver transplantation donor candidates to radiation. Acquiring CT images at a low $-\mathrm{kVp}$ could be a way to reduce the radiation exposure of potential donors who are usually young and therefore more radiosensitive and have longer life expectancies (24-27). Reducing the tube potential from 120 to $100 \mathrm{kVp}$ can reduce radiation exposure by about one third $(28,29)$. In contrast to tube current (mAs), a decrease in tube potential $(\mathrm{kVp})$ not only affects radiation exposure and noise levels, but also image contrast because $\mathrm{X}$-ray photons with higher energy can travel though the body without attenuation more easily than photons with lower energy potential. Lower proportion of Compton scattering compared to the photoelectric effect in X-ray photons of lower energy is another reason for a change in tissue contrast $(30,31)$. Therefore, $\mathrm{CT}$ attenuation values from low-kVp CT scans are different from those of $120-\mathrm{kVp} \mathrm{CT}$ scans and the reference range for CT attenuation values to evaluate hepatic steatosis in CT images obtained at a low $-\mathrm{kVp}$ may be different from the existing reference range for conventional $120-\mathrm{kVp}$ images.

In our study, the fat signal fraction was negatively correlated with MRS and CT attenuation indices. Both $\mathrm{CT}_{\mathrm{L}-\mathrm{S}}$ and $\mathrm{CT}_{\mathrm{L} / \mathrm{S}}$ provided high diagnostic performance for moderate to severe hepatic steatosis, with no significant difference between the two CT indices. Using a $\mathrm{CT}_{\mathrm{L}-\mathrm{S}}$ cut-off value of $-0.5 \mathrm{HU}$ and a $\mathrm{CT}_{\mathrm{L} / \mathrm{S}}$ cut-off value of 0.99 based on the Youden index to achieve a balance between sensitivity and specificity, both indices had $100 \%$ sensitivity and $84.46 \%$ specificity for diagnosing moderate to severe steatosis. When cut-off values of $6.9 \mathrm{HU}$ and 0.89 were used for $\mathrm{CT}_{\mathrm{L}-\mathrm{S}}$ and $\mathrm{CT}_{\mathrm{L} / \mathrm{S}}$, respectively, lower sensitivity (70.59-76.47\%) but higher specificity $(90.54 \%)$ were achieved compared to Youden index cut-off values.

Cut-off values of the Youden index reported in the previous study using $120-\mathrm{kVp}$ CT scans were $-7 \mathrm{HU}$ for $\mathrm{CT}_{\mathrm{L}-\mathrm{S}}$ and 0.9 for $\mathrm{CT}_{\mathrm{L} / \mathrm{S}}(17)$. In that study, the sensitivities and specificities for diagnosing moderate to severe fatty 
liver were $82 \%$ and $100 \%$ for both $\mathrm{CT}_{\mathrm{L}-\mathrm{S}}$ and $\mathrm{CT}_{\mathrm{L} / \mathrm{S}}$, respectively, representing a lower sensitivity and higher specificity than those estimated in our study. In another previous study using $120-\mathrm{kVp}$ scans, the sensitivities for diagnosing all degree steatosis and moderate to severe steatosis were $18.6 \%$ and $67 \%$, respectively, using a reference range cut-off value of $1 \mathrm{HU}$ for $\mathrm{CT}_{\mathrm{L}-\mathrm{S}}(18)$. Reference range of $\mathrm{CT}_{\mathrm{L}-\mathrm{S}}$ in that study was 1 to $18 \mathrm{HU}$ and much narrower than that in our study, -6.90 to $31.40 \mathrm{HU}$, and the standard deviation of that study $(4.2 \mathrm{HU})$ was much smaller than that in our study $(7.24 \mathrm{HU})$. We speculate that the wider range and greater standard deviation in our study than the previous study were due to the noisier images from low-kVp CT scans in our study and non-parametric characteristics of results of our study. Because of the lower cut-off value of $\mathrm{CT}_{\mathrm{L}-\mathrm{S}}$ in our study (-6.9 HU compared to $1 \mathrm{HU}$ ), sensitivity for any degree steatosis was higher in this study than that in the previous study using $120-\mathrm{kVp}$, but the sensitivity for moderate to severe steatosis was similar $(70.59 \%$ vs. $67 \%$, respectively). When using a cut-off value of $-6.9 \mathrm{HU}$ for $\mathrm{CT}_{\mathrm{L}-\mathrm{s}}$, our non-enhanced low-kVp CT protocol showed similar to superior diagnostic performance to that of $120-\mathrm{kVp}$ CT. Our low-kVp protocol can significantly reduce radiation exposure while allowing accurate diagnosis of hepatic steatosis. Furthermore, we only acquired a single slice unenhanced image covering both the liver and spleen to evaluate steatosis, which can minimize the radiation exposure of potential liver donors for LDLT.

In this study, we adopted multiecho MRS rather than liver biopsy as the reference standard. We thought this to be a reasonable strategy because multiecho MRS and MRI-PDFF have been demonstrated to be closely correlated to pathology results for evaluating hepatic steatosis and have been used as reference standards in recent clinical trials (13-15). Furthermore, this allowed us to avoid sampling errors and inter-observer variability, which are limitations of liver biopsy. We adopted cut-off values of the fat signal fraction for all degrees of steatosis $(\geq 4.14 \%)$ and moderate to severe steatosis $(\geq 15.72 \%)$ based on a study by Runge et al. (22) who used MRS with 3T MRI units. Also, other studies have reported similar cut-off values of the fat signal fraction for the diagnosis of hepatic steatosis in multiecho MRS and MRI-PDFF (12,32-35). Our MRI protocol includes in- and opposed-phase dual echo GRE technique and this also could be used for the quantification of hepatic fat deposition. However, dual echo imaging is mainly a qualitative tool and the measurement is less accurate than multiecho
MRS (36). Also, cut-off values for the degree of steatosis were not reported for dual echo imaging. Therefore, we adopted MRS as a standard reference.

There are several limitations to our study. First, we included mainly young and healthy adults because all of these individuals were potential candidates for LDLT. Therefore, only $10.3 \%$ of enrolled subjects had moderate to severe hepatic steatosis. To overcome this limitation, we used a reference range to calculate the optimal cutoff value to maximize specificity. Second, although CT attenuation values are not influenced by ethnic group, all subjects in our study were Asian adults. Furthermore, our data were collected from a single institute and therefore, further validation is mandatory to generalize our results. Third, we could not compare CT indices calculated from standard tube potential $(120-\mathrm{kVp})$ and low-kVp $(100-\mathrm{kVp})$ scans directly because we only acquired a single slice image using low $\mathrm{kV}$. However, we felt that it would be unethical to acquire CT images using both conventional and low-kVp in the same subjects for research purposes only, especially young and healthy subjects.

In conclusion, we found that $\mathrm{CT}$ indices measured from a low-kVp, single-slice unenhanced CT scan were negatively correlated with the degree of hepatic steatosis and provided high diagnostic performance for diagnosis of hepatic steatosis. Diagnostic performance of $\mathrm{CT}_{\mathrm{L}-\mathrm{S}}$ and $\mathrm{CT}_{\mathrm{L} / \mathrm{s}}$ were similar. CT indices from our low- $\mathrm{kVp}$, singleslice unenhanced CT protocol were robust for diagnosing moderate to severe hepatic steatosis and can help prevent unnecessary biopsies and minimize radiation exposure.

\section{Acknowledgments}

Funding: This work was supported by the National Research Foundation of Korea (NRF) grant funded by the Korea government (MSIT) (No. 2019R1F1A1060566). This work was also supported by the Technology Innovation Program (No. 20011856) funded By the Ministry of Trade, Industry \& Energy (MOTIE, Korea).

\section{Footnote}

Reporting Checklist: The authors have completed the STARD reporting checklist. Available at https://dx.doi. org/10.21037/qims-21-474

Conflicts of Interest: All authors have completed the ICMJE uniform disclosure form (available at https://dx.doi. 
org/10.21037/qims-21-474). JIC reports that he received grants from Guerbet Koreaand Samsung Medison. The other authors have no conflicts of interest to declare.

Ethical Statement: The authors are accountable for all aspects of the work in ensuring that questions related to the accuracy or integrity of any part of the work are appropriately investigated and resolved. The study was conducted in accordance with the Declaration of Helsinki (as revised in 2013). The study was approved by the institutional review board of the Catholic University of Korea (No. KC21RASI0263) and individual consent for this retrospective analysis was waived.

Open Access Statement: This is an Open Access article distributed in accordance with the Creative Commons Attribution-NonCommercial-NoDerivs 4.0 International License (CC BY-NC-ND 4.0), which permits the noncommercial replication and distribution of the article with the strict proviso that no changes or edits are made and the original work is properly cited (including links to both the formal publication through the relevant DOI and the license). See: https://creativecommons.org/licenses/by-nc-nd/4.0/.

\section{References}

1. Boyce CJ, Pickhardt PJ, Kim DH, Taylor AJ, Winter TC, Bruce RJ, Lindstrom MJ, Hinshaw JL. Hepatic steatosis (fatty liver disease) in asymptomatic adults identified by unenhanced low-dose CT. AJR Am J Roentgenol 2010;194:623-8.

2. Li J, Zou B, Yeo YH, Feng Y, Xie X, Lee DH, et al. Prevalence, incidence, and outcome of non-alcoholic fatty liver disease in Asia, 1999-2019: a systematic review and meta-analysis. Lancet Gastroenterol Hepatol 2019;4:389-98.

3. Younossi ZM, Koenig AB, Abdelatif D, Fazel Y, Henry L, Wymer M. Global epidemiology of nonalcoholic fatty liver disease-Meta-analytic assessment of prevalence, incidence, and outcomes. Hepatology 2016;64:73-84.

4. Eslam M, Newsome PN, Sarin SK, Anstee QM, Targher G, Romero-Gomez M, et al. A new definition for metabolic dysfunction-associated fatty liver disease: An international expert consensus statement. J Hepatol 2020;73:202-9.

5. Pickhardt PJ, Jee Y, O'Connor SD, del Rio AM. Visceral adiposity and hepatic steatosis at abdominal CT: association with the metabolic syndrome. AJR Am J Roentgenol 2012;198:1100-7.
6. Chen YS, Cheng YF, De Villa VH, Wang CC, Lin CC, Huang TL, Jawan B, Chen CL. Evaluation of living liver donors. Transplantation 2003;75:S16-9.

7. Pickhardt PJ, Park SH, Hahn L, Lee SG, Bae KT, Yu ES. Specificity of unenhanced CT for non-invasive diagnosis of hepatic steatosis: implications for the investigation of the natural history of incidental steatosis. Eur Radiol 2012;22:1075-82.

8. Meng H, Yang J, Yan L. Donor Safety in Adult-Adult Living Donor Liver Transplantation: A Single-Center Experience of 356 Cases. Med Sci Monit 2016;22:1623-9.

9. Veteläinen R, van Vliet A, Gouma DJ, van Gulik TM. Steatosis as a risk factor in liver surgery. Ann Surg 2007;245:20-30.

10. Lee JY, Kim KM, Lee SG, Yu E, Lim YS, Lee HC, Chung YH, Lee YS, Suh DJ. Prevalence and risk factors of nonalcoholic fatty liver disease in potential living liver donors in Korea: a review of 589 consecutive liver biopsies in a single center. J Hepatol 2007;47:239-44.

11. Idilman IS, Aniktar H, Idilman R, Kabacam G, Savas B, Elhan A, Celik A, Bahar K, Karcaaltincaba M. Hepatic steatosis: quantification by proton density fat fraction with MR imaging versus liver biopsy. Radiology 2013;267:767-75.

12. Middleton MS, Heba ER, Hooker CA, Bashir MR, Fowler KJ, Sandrasegaran K, Brunt EM, Kleiner DE, Doo E, Van Natta ML, Lavine JE, Neuschwander-Tetri BA, Sanyal A, Loomba R, Sirlin CB; NASH Clinical Research Network. Agreement Between Magnetic Resonance Imaging Proton Density Fat Fraction Measurements and PathologistAssigned Steatosis Grades of Liver Biopsies From Adults With Nonalcoholic Steatohepatitis. Gastroenterology 2017;153:753-61.

13. Boudinaud C, Abergel A, Joubert-Zakeyh J, Fontarensky M, Pereira B, Chauveau B, Garcier JM, Chabrot P, Boyer L, Magnin B. Quantification of steatosis in alcoholic and nonalcoholic fatty liver disease: Evaluation of four MR techniques versus biopsy. Eur J Radiol 2019;118:169-74.

14. Caussy C, Reeder SB, Sirlin CB, Loomba R. Noninvasive, Quantitative Assessment of Liver Fat by MRI-PDFF as an Endpoint in NASH Trials. Hepatology 2018;68:763-72.

15. Noureddin M, Lam J, Peterson MR, Middleton M, Hamilton G, Le TA, Bettencourt R, Changchien C, Brenner DA, Sirlin C, Loomba R. Utility of magnetic resonance imaging versus histology for quantifying changes in liver fat in nonalcoholic fatty liver disease trials. Hepatology 2013;58:1930-40.

16. van Werven JR, Marsman HA, Nederveen AJ, Smits 
NJ, ten Kate FJ, van Gulik TM, Stoker J. Assessment of hepatic steatosis in patients undergoing liver resection: comparison of US, CT, T1-weighted dual-echo MR imaging, and point-resolved 1H MR spectroscopy. Radiology 2010;256:159-68.

17. Park SH, Kim PN, Kim KW, Lee SW, Yoon SE, Park SW, Ha HK, Lee MG, Hwang S, Lee SG, Yu ES, Cho EY. Macrovesicular hepatic steatosis in living liver donors: use of CT for quantitative and qualitative assessment. Radiology 2006;239:105-12.

18. Park YS, Park SH, Lee SS, Kim DY, Shin YM, Lee W, Lee SG, Yu ES. Biopsy-proven nonsteatotic liver in adults: estimation of reference range for difference in attenuation between the liver and the spleen at nonenhanced CT. Radiology 2011;258:760-6.

19. Fritz GA, Schoellnast H, Deutschmann HA, Wiltgen M, Brader P, Berghold A, Groell R. Density histogram analysis of unenhanced hepatic computed tomography in patients with diffuse liver diseases. J Comput Assist Tomogr 2006;30:201-5.

20. Limanond P, Raman SS, Lassman C, Sayre J, Ghobrial RM, Busuttil RW, Saab S, Lu DS. Macrovesicular hepatic steatosis in living related liver donors: correlation between CT and histologic findings. Radiology 2004;230:276-80.

21. Bydder M, Yokoo T, Hamilton G, Middleton MS, Chavez $\mathrm{AD}$, Schwimmer JB, Lavine JE, Sirlin CB. Relaxation effects in the quantification of fat using gradient echo imaging. Magn Reson Imaging 2008;26:347-59.

22. Runge JH, Smits LP, Verheij J, Depla A, Kuiken SD, Baak BC, Nederveen AJ, Beuers U, Stoker J. MR Spectroscopy-derived Proton Density Fat Fraction Is Superior to Controlled Attenuation Parameter for Detecting and Grading Hepatic Steatosis. Radiology 2018;286:547-56.

23. Weinstein S, Obuchowski NA, Lieber ML. Clinical evaluation of diagnostic tests. AJR Am J Roentgenol 2005;184:14-9.

24. Guimarães LS, Fletcher JG, Harmsen WS, Yu L, Siddiki H, Melton Z, Huprich JE, Hough D, Hartman $\mathrm{R}, \mathrm{McCollough} \mathrm{CH}$. Appropriate patient selection at abdominal dual-energy CT using $80 \mathrm{kV}$ : relationship between patient size, image noise, and image quality. Radiology 2010;257:732-42.

25. Funama Y, Awai K, Nakayama Y, Kakei K, Nagasue N, Shimamura M, Sato N, Sultana S, Morishita S, Yamashita Y. Radiation dose reduction without degradation of lowcontrast detectability at abdominal multisection CT with a low-tube voltage technique: phantom study. Radiology
2005;237:905-10.

26. Royal HD. Effects of low level radiation-what's new? Semin Nucl Med 2008;38:392-402.

27. Seyal AR, Arslanoglu A, Abboud SF, Sahin A, Horowitz JM, Yaghmai V. CT of the Abdomen with Reduced Tube Voltage in Adults: A Practical Approach. Radiographics 2015;35:1922-39.

28. Raman SP, Johnson PT, Deshmukh S, Mahesh M, Grant KL, Fishman EK. CT dose reduction applications: available tools on the latest generation of CT scanners. J Am Coll Radiol 2013;10:37-41.

29. Sigal-Cinqualbre AB, Hennequin R, Abada HT, Chen X, Paul JF. Low-kilovoltage multi-detector row chest CT in adults: feasibility and effect on image quality and iodine dose. Radiology 2004;231:169-74.

30. Martin C. The importance of radiation quality for optimisation in radiology. Biomed Imaging Interv $\mathbf{J}$ 2007;3:e38.

31. Seibert JA. Tradeoffs between image quality and dose. Pediatr Radiol 2004;34 Suppl 3:S183-95; discussion S234-41.

32. Imajo K, Kessoku T, Honda Y, Tomeno W, Ogawa Y, Mawatari H, Fujita K, Yoneda M, Taguri M, Hyogo H, Sumida Y, Ono M, Eguchi Y, Inoue T, Yamanaka T, Wada K, Saito S, Nakajima A. Magnetic Resonance Imaging More Accurately Classifies Steatosis and Fibrosis in Patients With Nonalcoholic Fatty Liver Disease Than Transient Elastography. Gastroenterology 2016;150:626637.e7.

33. Park CC, Nguyen P, Hernandez C, Bettencourt R, Ramirez K, Fortney L, Hooker J, Sy E, Savides MT, Alquiraish MH, Valasek MA, Rizo E, Richards L, Brenner D, Sirlin CB, Loomba R. Magnetic Resonance Elastography vs Transient Elastography in Detection of Fibrosis and Noninvasive Measurement of Steatosis in Patients With Biopsy-Proven Nonalcoholic Fatty Liver Disease. Gastroenterology 2017;152:598-607.e2.

34. Tang A, Desai A, Hamilton G, Wolfson T, Gamst A, Lam J, Clark L, Hooker J, Chavez T, Ang BD, Middleton MS, Peterson M, Loomba R, Sirlin CB. Accuracy of MR imaging-estimated proton density fat fraction for classification of dichotomized histologic steatosis grades in nonalcoholic fatty liver disease. Radiology 2015;274:416-25.

35. Tang A, Tan J, Sun M, Hamilton G, Bydder M, Wolfson T, Gamst AC, Middleton M, Brunt EM, Loomba R, Lavine JE, Schwimmer JB, Sirlin CB. Nonalcoholic fatty liver disease: MR imaging of liver proton density fat fraction to 
assess hepatic steatosis. Radiology 2013;267:422-31.

36. Lee SS, Lee Y, Kim N, Kim SW, Byun JH, Park SH, Lee MG, Ha HK. Hepatic fat quantification using chemical shift MR imaging and MR spectroscopy in the presence of hepatic iron deposition: validation in phantoms and in patients with chronic liver disease. J Magn Reson Imaging 2011;33:1390-8.

Cite this article as: Choi Y, Kim DK, Youn SY, Kim H, Choi JI. Unenhanced computed tomography for non-invasive diagnosis of hepatic steatosis with low tube potential protocol. Quant Imaging Med Surg 2022;12(2):1348-1358. doi: 10.21037/ qims-21-474 